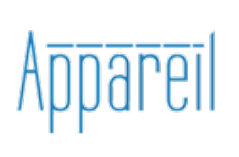

Appareil

$6 \mid 2010$

Philosophie et cinéma

\title{
Le cinéma, un appareil de déviance : N. Philibert
}

Journée : Moi, Pierre Rivière de R.Allio, le 31 janvier 2008

Jean-Louis Déotte

(2) OpenEdition

Journals

Édition électronique

URL : http://journals.openedition.org/appareil/277

DOI : 10.4000/appareil.277

ISSN : 2101-0714

Éditeur

MSH Paris Nord

Référence électronique

Jean-Louis Déotte, «Le cinéma, un appareil de déviance : N. Philibert », Appareil [En ligne], 6 | 2010, mis en ligne le 15 mars 2008, consulté le 30 juillet 2020. URL : http://journals.openedition.org/appareil/277 ; DOI : https://doi.org/10.4000/appareil.277

Ce document a été généré automatiquement le 30 juillet 2020.

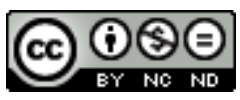

Appareil est mis à disposition selon les termes de la Licence Creative Commons Attribution - Pas d'Utilisation Commerciale - Pas de Modification 4.0 International. 


\section{Le cinéma, un appareil de déviance : N. Philibert}

Journée : Moi, Pierre Rivière de R.Allio, le 31 janvier 2008

Jean-Louis Déotte

1 N. Philibert a travaillé avec R. Allio pour le tournage de Moi, Pierre Rivière qui ai égorgé ma mère, mon frère, ma sœur (1976) et revient dans Retour en Normandie (2007) sur les lieux du tournage pour retrouver les acteurs. Tous sont présents, sauf Claude Hébert qui avait joué le rôle de Pierre Rivière et qui reste énigmatiquement absent.

2 Il n'a pas été possible de tourner sur les lieux mêmes de la famille Rivière, mais un peu plus loin, toujours aux confins de l'Orne et du Calvados.

3 C'est une région de bocage, de fortes traditions paysannes, apparemment, mais seulement apparemment, ouvertes à l'étranger. Pour le Cotentin, les habitants de la presqu'île ont forgé le terme de horsain pour dire cette altérité irréductible.

4 C'est dire que l'arrivée de l'équipe d'Allio a dû susciter beaucoup de méfiance, l'équipe a dû démarcher de ferme en ferme pour contacter les acteurs, en particulier les jeunes filles soumises à l'autorité parentale. C'est pour cette raison que leur témoignage est exceptionnel: là où il $\mathrm{y}$ avait de fortes probabilités pour que les uns et les autres n'échappent pas au schéma de la reproduction sociale au sens de Bourdieu, l'irruption de l'appareil cinématographique au sens large, pas seulement l'objet technique (la caméra considérée dans son individualité), mais l'ensemble technique : l'équipe venue de Paris, le chariot du travelling et ses rails, la grue, les projecteurs, en amont la production et le synopsis, en aval, toute la distribution, la communication, bref tout cela a généré un milieu associé nouveau, ce que Benjamin appelle un collectif.

5 Je voudrais m'arrêter sur ce milieu associé (les acteurs du film d'Allio, aussi bien que les spectateurs) qui déborde largement l'application d'un appareil à un milieu social « immédiat ». J'entends par appareil bien plus qu'un dispositif qui, selon Foucault ou Agamben, ne lierait que les séries du pouvoir et du savoir, l'exemple princeps étant celui du panopticon de Surveiller et punir. L'appareil est plus que le dispositif technique, car c'est ce qui va configurer l'apparition de l'apparaître et pour cela va faire époque. 
Un exemple simple : l'« outil visuel » qu'utilise Buren, à savoir l'alternance de bandes horizontales blanches et noires comme au Palais Royal à Paris, de couleurs ailleurs, cet outil est un appareil parce qu'il fait surgir du spectacle urbain ce que l'on n'avait jamais observé attentivement : tel ou tel édifice, en l'occurrence, le Palais Royal, un des lieux les plus complexes de Paris. Ou à l'intérieur d'un musée, l'ensemble des cadres de tableau et des cimaises dont la fonction de suspension, est certes de s'effacer devant l'œuvre, mais, qui, comme à Reims, peuvent être eux-mêmes mis en exergue comme ce qui mettait en valeur la peinture, etc. L'appareil rend visible ce qui sans lui aurait disparu dans un flux de perception, selon la formule de K. Fiedler le maître de P. Klee. L'appareil est anti-bergsonien, et pour une autre raison aussi: c'est qu'il est nécessairement technique (ici l'alternance minimale des couleurs sur des bandes de $8,7 \mathrm{~cm}$ chacune).

6 J'emprunte les termes de «milieu naturel ou milieu immédiat » et « milieu associé » à Simondon, mais il est évident que dans le cas qui nous intéresse, le milieu paysan bocager bas-normand n'a rien de naturel, c'est déjà un milieu associé, du fait de l'intervention antérieure d'autres appareils comme le récit qui transmet la tradition païenne ou comme l'Église qui fabrique un corps d'inclusion christique à partir de la Révélation. Retour en Normandie porte témoignage du fait que l'irruption de l'appareil cinématographique à un certain moment historique en un certain lieu géographique et social a introduit une déviance dans l'état antérieur du milieu. C'est que les appareils antérieurs, narratifs ou d'incorporation christique, avaient introduit selon leur mode des temporalités spécifiques, idéalement l'attente du Jugement dernier pour les Chrétiens par exemple, et que ces temporalités enchâssées les unes dans les autres, déterminaient les communautés paysannes selon la figure du destin, et déjà du destin social. Le témoignage des acteurs de Philibert est à ce titre révélateur: les uns et les autres se destinaient à occuper le rôle social qui les attendait, de tous temps, comme les paysans béarnais décrits par Bourdieu. La scène d'ouverture est cohérente avec cette succession de ruptures, l'éleveur brutalise un porcelet tout juste mis à bât, mais c'est pour le faire sortir du coma post-natal. Pour les acteurs amateurs du film d'Allio, la participation au tournage de Pierre Rivière constitua le moment le plus important de leur vie. Ce fut une scansion : il y a un avant et un après. Les choix professionnels et politiques sont là pour le rappeler : un tout autre regard sur la maladie mentale de ces parents d'une enfant schizophrène, les soins donnés à des sujets débiles mentaux dans une institution pour cette femme qui aurait dû travailler la terre, la participation, dans ce pays fortement conservateur, à la lutte antinucléaire de cette boulangère qui par ailleurs lutte contre une aphasie. Et surtout, pour Claude Hébert, un autre film ( $\mathrm{La}$ Drôlesse de Doillon), le départ à Paris comme acteur puis la conversion et l'installation à Haïti auprès des populations les plus démunies, pour celui qui jouait Pierre Rivière et qui revient dans le film de Philibert comme prêtre. Claude Hébert déclare aujourd'hui qu'il était d'une grande timidité et qu'il avait voulu faire aimer Pierre Rivière. Il voit dans sa vocation une continuité avec le travail de comédien : la nécessité de témoigner.

7 Il me semble que bien des films de Philibert soutiennent cette politique: dans La moindre des choses (1996), les malades de la clinique psychiatrique de La Borde, haut lieu de l'anti-psychiatrie française et de l'analyse anti-institutionnelle, héritier de Saint Alban en Lozère et de Tosquelles, montent avec l'aide du personnel une pièce de Gombrowicz : Opérette. On sait que le personnel de la clinique est très polyvalent et que son rôle réel peut prêter à confusion : le visiteur ne peut savoir qui est en traitement et qui travaille dans l'institution puisque les apparences sont les mêmes, la rotation des 
tâches étant la règle. Il s'agit donc de filmer le spectacle théatral mis en œuvre par les patients lors de la traditionnelle fête du 15 août où les familles vont constituer le public. L'important, c'est la rupture entre le long et pénible travail de répétitions et la "première" devant un public de familiers : autant l'apprentissage d'un texte ou d'un air musical est difficile, voire semble impossible du fait du mutisme de la personne, parce que l'apprentissage du rôle consiste en une lutte avec le délire qui enferme dans une répétition létale, autant la "première" qui est un acte collectif de représentation, métamorphose les uns et les autres. C'est sur ce renversement que je voudrais m'arrêter en analysant les dispositifs de spectacle (théâtre et cinéma) comme des appareils anti-destin. Les autres documentaires de Philibert (Un animal, des animaux, La ville Louvre, etc.) mettant plutôt en valeur les sans-grade de l'institution muséale, comme le taxidermiste du Museum d'histoire naturelle: Jacky Thiney, plutôt que les conservateurs et autres muséographes. La pratique de Philibert consiste à arriver sans scénario dans un lieu, sans idée esthétique à incarner comme l'aurait fait l'idéalisme allemand ou Brecht (Benjamin), à mettre en confiance ceux qui vont devenir ses partenaires et à les laisser travailler. Puis l'interview de certains permet de centrer le film sur une véritable personnalité. Le risque est évident : transformer celui qui donne librement ses apparences en acteur d'un jour ne comprenant pas pourquoi la gloire lui échapperait (le procès d'être et Avoir).

8 On sait que Bourdieu a consacré son œuvre à la description des dispositifs sociaux de reproduction, un habitus étant toujours le produit d'une éducation, qui elle-même redéveloppe en boucle cet habitus, et la marge du jeu individuel étant toujours limitée à ce que permet l'institution. De ce point de vue, l'irruption d'un nouvel appareil va toujours, au moins dans un premier temps, contre la reproduction.

9 La consistance du milieu associé spécifique qu'a forgé le film d'Allio confirme à une échelle expérimentale les thèses sur le cinéma développées par Benjamin dans L'œuvre d'art à l'époque de sa reproductibilité technique. Je ne peux pas revenir sur l'analyse de ces thèses que j'ai développée dans L'époque des appareils, mais je peux rappeler néanmoins que pour lui le cinéma a généré un nouveau droit politique : le droit de donner de soimême ses apparences en un moment qu'il caractérise comme étant celui du test généralisé, c'est-à-dire celui de l'observation objectivante du prolétariat (ou de la jeunesse ou des sportifs professionnels) par les maitres du monde. Ce faisant Benjamin avait répondu par avance à Foucault : certes un dispositif visuel peut servir à contrôler une population donnée, l'enjeu c'est donc pour elle de le retourner en appareil. Je voudrais commenter un autre texte assez méconnu de Benjamin, destiné à conforter le travail pédagogico-politique de son amie bolchévique Asja Lacis auprès de hordes d'enfants abandonnés dans les années vingt errant dans un grand nombre de villes russes dévastées par la guerre civile. Le texte de Benjamin de 1928 se trouve inséré dans les mémoires d'A. Lacis : Profession: Révolutionnaire. Sur le théâtre prolétarien. Meyerhold, Brecht, Benjamin, Piscator traduit par Ph. Ivernel et publié aux PUG en 1989.

D'après le texte de Benjamin, qui est un court manifeste sur le théâtre prolétarien pour enfants, lequel n'est donc pas un théâtre d'idée, qualifié pour cela d'«idéaliste allemand », le milieu associé est constitué comme un collectif d'enfants travaillant avec l'aide d'animateurs adultes, lesquels de formateurs vont devenir formés en apprenant à lire grâce aux enfants. Il y aura donc un double renversement: les enfants assujettis à leur destin d'enfants des rues vont produire activement une sorte d'écriture de signaux corporels à déchiffrer, les adultes devenant leurs lecteurs. Pour Benjamin, ce double 
renversement est proprement carnavalesque, ce qui veut dire chez lui, révolutionnaire :

La représentation est la grande pause créatrice dans l'œuvre d'éducation. Elle est au royaume des enfants l'équivalent du carnaval dans les cultes anciens. Elle offre l'image du monde renversé : de même qu'à Rome, durant les Saturnales, le maître servait l'esclave, les enfants, eux, le temps d'une représentation, occupent la scène pour l'instruction et pour l'éducation des éducateurs attentifs. De nouvelles innervations, de nouvelles énergies se manifestent, dont l'animateur n'avait souvent rien deviné en cours de travail. Il apprend à les connaître dans l'émancipation tumultueuse de l'imagination enfantine. (p. 57)

C'est dire que ces animateurs doivent au moment de la «première » se retirer, devenir eux-mêmes spectateurs, ayant à déchiffrer des signaux émis par ces jeunes acteurs. Pour Benjamin, on est strictement dans le monde de la représentation, la "première " est bien une représentation où se donne toute la vitalité de la jeunesse. La représentation est donc première, elle ne vient pas succéder à une présence ou à un texte déjà écrit dont elle serait la présentation sur scène. Elle a toute la légitimité et toute l'autorité d'un acte fondateur rendant possible toute une série de variations où, selon lui, excellent les enfants. Ce qui rend possible l'expression de l'improvisation sous la forme de signaux, c'est un cadre préalable, celui du théâtre. Que Benjamin refuse de définir autrement que comme un système ou un champ objectif limité rendant possible l'éducation de l'enfant, laquelle « exige que sa vie entière soit mise en jeu » (p. 51). Ce qui caractérise aussi les acteurs amateurs de R. Allio. Dans ce sens, le théâtre de représentation où le jeu ne se distingue pas de la réalité, écrit-il, est bien un appareil qui traite de certaines apparences, les signaux corporels délivrés par les enfants pour les transformer en texte et en actions dont la vérité engage l'avenir.

Il faut s'arrêter sur ce type de signe : comme la représentation est surtout affaire de tensions, de résolution de tensions, de champ de forces, on peut penser qu'il y va d'une intensité, d'une énergétique plus que d'une rhétorique. Les signaux exprimés par les collectifs d'enfants sont de l'ordre de l'imagination enfantine, toujours magique et pour cela dangereuse pour les protagonistes eux-mêmes (p. 54). La tâche des animateurs consiste à innerver autrement le jeu des enfants sur le modèle du travail du peintre qui innerve l'œil par la main quand l'œil commence à se paralyser selon la référence à Fiedler (Écrits sur l'art).

Le peintre n'est pas un individu qui aurait une vision plus naturaliste, plus poétique ou plus extatique que d'autres, mais plutôt un homme sachant regarder de plus près avec la main quand l'œil se paralyse, et qui transfère l'innervation réceptive des muscles de la vue dans l'innervation créatrice de la main. Mise en rapport exacte de l'innervation créatrice avec la réceptive, tel est le geste enfantin. (p. 54)

Pour Benjamin, l'enchaînement sur cette innervation doit être le fait des autres formes d'expressions improvisées: peinture, récitation, musique, danse. Le passage de la simple vision, qui voit de loin, comme dans la contemplation d'un tableau, à une perception quasi tactile sera au cœur de l'analyse du cinéma (L'œuvre d'art) où il comparera perception de l'architecture et perception d'un film, puisque dans les deux cas, l'attention ne peut plus être mobilisée (le flâneur parcourant une ville ne prête pas attention aux édifices, on dira que sa perception est de balayage, non focalisante, c'està-dire de distraction, de même pour un film). En 1928 Benjamin n'a pas encore écrit sur le cinéma, mais déjà il caractérise la forme de perception la plus appropriée à l'esthétique moderne, qui est une esthétique du choc, et qui en même temps délivre des "dangereuses magies de la pure imagination », en déclarant que c'est une perception 
qui doit « donner pouvoir exécutif sur les matériaux» (p. 54), c'est donc une perception tactile où l'innervation de la main relève celle, défaillante, de l'œil. C'est la raison pour laquelle, analysant le cinéma après la photographie, Benjamin fera un bon accueil à la question de la technique, puisque pour lui l'appareil cinématographique est dans le prolongement de cette relève du visuel par la main technicienne qui a effrayé bien de nos philosophes contemporains depuis Heidegger. Certes, les propositions de Benjamin sont proches des tentatives des groupes Medvedkine, mais si elles concernent aussi notre rapport à la ville actuelle, ce rapport peut être certes de distraction tout en ayant une visée pratique. La ville n'est pas pour lui un spectacle comme le serait un film de divertissement, mais l'occasion d'interventions pratiques. C'est peut-être le seul moyen de donner un sens à la politique situationniste de la ville qui sinon, reste superficielle.

Philibert aura saisi le milieu associé généré par le film d'Allio au moment où ce milieu, grâce à la représentation, a rompu avec son destin, et étant de fait suspendu, est devenu capable de se prendre en main. L'intervention de l'appareil cinématographique a rompu le charme du récit traditionnel et du corps communautaire: ce sont des normes de légitimité constitutives de l'être-ensemble qui sont devenues obsolètes. Au profit de la nouvelle norme de légitimité : la norme délibérative. Le cas «Rivière » rend intelligible l'imposition de cette norme dans les années $30 \mathrm{du}$ xIX ${ }^{e}$ siècle, quand le débat entre juristes et aliénistes porte sur la responsabilité pénale de l'inculpé. Débat affirmatif puisqu'il débouchera sur sa reconnaissance dans le code pénal.

\section{BIBLIOGRAPHIE}

Asja Lacis, Profession : Révolutionnaire. Sur le théâtre prolétarien. Meyerhold, Brecht, Benjamin, Piscator, Ph. Ivernel (trad.), Éd. Presses universitaires de Grenoble, 1989.

Benjamin Walter, «L'œuvre d'art à l'époque de sa reproductibilité technique » (dernière version de 1939), in Euvres III, Maurice de Gandillac (trad.), revue par Rainer Rochlitz, Paris, Gallimard, 2000, p. 269-316.

\section{RÉSUMÉS}

N. Philibert avait été l'assistant de R. Allio pour le tournage du film tiré des travaux de l'équipe réunie par Foucault autour $\mathrm{du}$ "cas» Pierre Rivière. Son film effectue un "Retour en Normandie » pour découvrir le devenir des acteurs amateurs du film d'Allio. Le tournage aura été un véritable clinamen dans leur vie. Philibert retrouve en fait, sans le savoir, ce que Benjamin dit du théâtre prolétarien pour enfants inauguré par A. Lascis : être en représentation, c'est libérer de nouvelles énergies comme dans le moment du carnaval. C'est une étape que d'autres auraient qualifiée rapidement de « révolutionnaire ». 
INDEX

Personnes citées : Benjamin (Walter), Allio (René), Foucault (Michel), Lacis (Asja)

Mots-clés : Moi Pierre Rivière, innervation, représentation, théâtre soviétique

\section{AUTEUR}

JEAN-LOUIS DÉOTTE

Professeur des universités (philosophie esthétique), département de philosophie Paris 8 